Research Article

\title{
The Reduction of Modal Sensor Channels through a Pareto Chart Methodology
}

\author{
Kaci J. Lemler and William H. Semke \\ Mechanical Engineering, University of North Dakota, Stop 8359, Grand Forks, ND 58202, USA \\ Correspondence should be addressed to William H. Semke; william.semke@engr.und.edu
}

Received 7 April 2015; Revised 5 May 2015; Accepted 16 June 2015

Academic Editor: Guiyun Tian

Copyright ( 2015 K. J. Lemler and W. H. Semke. This is an open access article distributed under the Creative Commons Attribution License, which permits unrestricted use, distribution, and reproduction in any medium, provided the original work is properly cited.

\begin{abstract}
Presented herein is a new experimental sensor placement procedure developed to assist in placing sensors in key locations in an efficient method to reduce the number of channels for a full modal analysis. It is a fast, noncontact method that uses a laser vibrometer to gather a candidate set of sensor locations. These locations are then evaluated using a Pareto chart to obtain a reduced set of sensor locations that still captures the motion of the structure. The Pareto chart is employed to identify the points on a structure that have the largest reaction to an input excitation and thus reduce the number of channels while capturing the most significant data. This method enhances the correct and efficient placement of sensors which is crucial in modal testing. Previously this required the development and/or use of a complicated model or set of equations. This new technique is applied in a case study on a small unmanned aerial system. The test procedure is presented and the results are discussed.
\end{abstract}

\section{Introduction}

Modal analysis is the study of the vibration modes and natural frequencies of a structure and is essential to a full understanding of a structure's vibration characteristics. Channel reduction is an important topic in modal analysis. With every extraneous channel there are associated costs. These costs include an extra sensor and the time spent installing, indexing, and logging the sensor as well as processing and analyzing the data obtained from said sensor [1]. Because of this, it is important to use as few channels as possible while still keeping enough to identify the modes of the structure [2-4]. A key point in channel reduction is sensor location selection.

Several methods for identifying important locations for sensors have been developed. One common method is to use a finite element model in which sensor sets are found which maximize the ability to observe modes while constraining each sensor to contribute unique information [5]. Another method selects sensor locations that make the corresponding target mode shape partitions as linearly independent as possible while maximizing the signal strength of the target modal responses within the sensor data [6]. Several methods use a genetic algorithm methodology to identify sensor locations by starting with a relatively small number of possible final locations and evolving these locations to the best set [7-11]. There is also a method that locates sensors at the maximum response position of an orthogonal sequence of vectors [12]. An efficient computational method to create an optimal sensor configuration using a Pareto approach has been demonstrated to be effective in structural identification [13].

Optimizing modal analysis techniques has been, and continues to be, a very active field of study within the structural dynamics community. In addition to those previously mentioned, earlier work addressing optimal sensor placement was done by Kammer, Kirkegaard and Brincker, Penny et al., Shah and Udwadia, and Udwadia [14-18]. In the field of structural damage detection, sensor placement methodologies are critical to success and several methods have been proven useful in studies by Cobb and Liebst, Heredia-Zavoni et al., Hemez and Farhat, Shi et al., Sohn and Law, Rao et al., and Yi et al. [19-25]. Structural health monitoring also requires sensor placement strategies that have been studied by Yi et al., Li et al., and Debnath et al. [26-29]. Bayesian probabilistic approaches have been shown to be effective in determining the optimal sensor configuration based on model estimations by Beck and Katafygiotis, Beck and Yuen, Katafygiotis et al., Papadimitriou et al., and Yuen and Kuok [30-34]. These 
researchers and many others have made significant contributions in the field and there are several more methods for sensor location identification. However, the majority require extensive finite element modeling, algorithm development, mathematical modeling, or a combination thereof. While the previous research is an excellent resource for modal sensor placement research, a fully experimental procedure that uses a statistical methodology to identify sensor locations that have the most significance in a modal analysis has been lacking and is absent in the literature. The method presented here helps fill this void with an experimental procedure that can reduce the number of channels of data collected while still capturing the primary modes of interest. This relatively quick and easy, noncontact, experimental method with which to identify important sensor locations for existing complex systems that are difficult and time consuming to model is a novel technique leveraging statistical analysis tools with contemporary vibration measurement techniques.

The method presented here uses a Pareto chart to identify important sensor locations from an initial experimental test set, thus eliminating extensive structural modeling. A laser vibrometer is a suitable tool to use to acquire the initial measurements of the candidate set of locations because of its mobility and its capability to gather vibration data in a noncontact manner. Laser vibrometers measure surface motion using the Doppler shift concept to measure the velocity of surface vibration [35]. Multipoint laser vibrometers have been used for several years for modal analysis, but they have not been used for quickly and efficiently identifying important sensor locations from an initial candidate set. A test was performed in which a single beam laser vibrometer was used to measure the velocity at several locations of the surface of a structure under excitation. These values were then used to identify locations of high interaction.

The most important sensor locations were identified from the laser vibrometer values using a Pareto chart. A Pareto chart is a statistical tool that can be utilized to identify the variables that are the most significant. The use of a Pareto chart to identify the most significant and critical sensor locations for modal analysis instrumentation is illustrated through a case study on a small unmanned aerial system (UAS).

\section{Method Theory}

The Pareto principle, which states that $80 \%$ of the effects are caused by $20 \%$ of the causes, was based on the observation by Pareto that $80 \%$ of the wealth in Italy belonged to $20 \%$ of the population [36]. This principle led to the introduction of Pareto charts in the field of quality control. It serves as a method to concentrate efforts on the factors of the greatest influence or impact. The ability to identify and concentrate efforts on a relatively small set of factors from an initially large set is crucial for efficient operations. This tool has been used in many different fields including identification of the most effective plasma processing variable that would impact the porosity of an aluminum oxide coating and for a sustainability root cause analysis method for chemical/energy production systems $[37,38]$. The Pareto chart was originally developed for use in the field of economics, and studies employing the Pareto chart can be found in the fields of natural and social sciences as well as physical sciences [39]. A Pareto chart is a vertical bar graph in which the relative frequency of each of the events is plotted in decreasing order from left to right. A line, representing the cumulative total, is then plotted on top of the bars. Pareto charts are used to determine the most significant aspects of a body of information by quickly and easily identifying which elements have the most effect [40]. Resources can then be used on the important aspects and not wasted on trivial aspects.

The structural modes and natural frequencies of a structure can be obtained through ground vibration tests (GVTs). The main tool used in a GVT is the frequency response function (FRF). This function can be based on the displacement, velocity, or acceleration response of a system to an applied force [41]. The expression for any FRF can be written in its general form as

$$
H_{j k}(\omega)=\frac{X_{j}}{F_{k}}=\sum_{r=1}^{N} \frac{{ }^{A} A_{j k}}{\lambda_{r}^{2}-\omega^{2}},
$$

where $X_{j}$ is the harmonic response in one of the degrees of freedom, $j$, caused by $F_{k}$, which is a harmonic force at a different degree of freedom, $k ;{ }_{r} A_{j k}$ is the modal constant (relative displacement at that DOF during vibration of the $r$ th mode); $\lambda_{r}^{2}$ is the eigenvalue of the $r$ th mode; $\omega$ is the frequency; and $N$ is the number of modes. Modal analysis is performed by curve-fitting the FRF obtained from the testing to obtain modes and then applying that data to a model of the structure to find the associated mode shapes. A complete description of modal testing can be found in Ewins [42].

The recommended testing procedure when using the sensor location identification method is outlined as follows. First, the structure should be mounted in a test rig so that it is in the desired modal configuration for the modal test. This can be free-free, fixed, or different boundary conditions. An excitation source and type then need to be decided upon and installed. This could be a shaker with random or sinusoidal excitation, an impact hammer, or other such device. The same excitation device should be used for both the sensor location identification method and the modal test. The excitation device should also be installed in the same location and direction for both tests. The location(s) should be such that all of the modes of the structure are excited. Next, a grid pattern needs to be laid out on the structure at which to take measurements. The density and locations of the grid points need to be decided upon carefully as this is a critical part of the test. The density, especially, can affect the results of the sensor location identification test. All of the potential sensor locations can then be quickly and efficiently scanned for a full structure evaluation. This is accomplished by taking and recording measurements of the motion at each of the grid points. This data can then be processed to produce the RMS values of the voltage at each point. A laser vibrometer is a good tool to use in this step as it is a quick, noncontact way of obtaining measurements, but other tools, such as an accelerometer, can be used as well. The next step is to construct a Pareto chart to see which of the 
measured locations experiences the largest relative amplitude under the excitation. An appropriate Pareto level then needs to be decided upon to identify the cutoff point in the Pareto chart. This information is then used to identify the significant locations at which to place sensors. These significant locations can then be instrumented with accelerometers and a full modal analysis can be performed. The modal analysis yields FRFs from which the natural frequencies and mode shapes can be extracted. These values can then be analyzed to understand the modal characteristics of the structure. In summary, the basic procedure for implementing the sensor placement technique using a Pareto chart is as follows:

(1) Install the test specimen in an appropriate mount for the test conditions desired.

(2) Create a grid pattern of candidate sensor locations.

(3) Excite the structure and record the dynamics response at each candidate position.

(4) Calculate the RMS amplitude at all positions.

(5) Construct a Pareto chart of all the candidate positions' RMS values.

(6) Establish an appropriate Pareto level that captures the desired structural responses.

(7) Instrument the reduced sensor set.

(8) Conduct a complete modal analysis of the structure.

\section{Test Description}

Several tests were performed using the sensor location identification method at different levels of channel reduction and the results were compared to a base test to analyze which setups still captured all of the necessary information. The tests were performed on the Super Hauler UAS produced by Bruce Tharpe Engineering. The Super Hauler is constructed of plywood, balsa wood, and Monokote and has a $3.7 \mathrm{~m}$ wingspan and a dry weight of $22 \mathrm{~kg}$ [43]. As outlined in the basic procedure, Step 1 was to isolate the Super Hauler by suspending it on bungee cords in a test rig so that all the wheels were $3.2 \mathrm{~cm}$ off the ground. This setup simulates a free-free boundary condition for modal testing [44]. The instrumented Super Hauler in the test rig can be seen in Figure 1.

The laser vibrometer that was used for the tests was a Polytech OFV 2601 Laser Vibrometer Controller with a Polytech CLV Laser Unit and a Polytech CLV 700 Laser Head. The laser vibrometer was mounted on a stand and directed to measure the vibration of the aircraft, as shown in Figure 2. The candidate locations for preliminary testing were chosen (Step 2) and are shown in Figure 3. All measurements were in the $Z$ direction except for the two on the vertical stabilizer which were in the $Y$ direction.

Step 3 was to excite the Super Hauler using a small shaker and collect the data. The shaker used was the Mini SmartShaker with an integrated power amplifier from The Modal Shop Inc. The shaker was attached to the aircraft

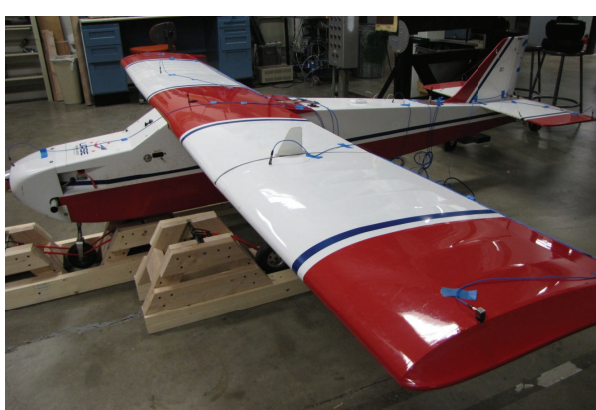

FIGURE 1: The Super Hauler in the test rig while instrumented with accelerometers.

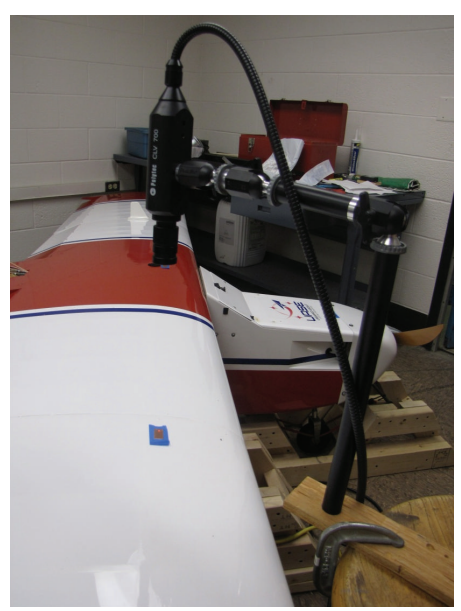

FIGURE 2: Test setup with the laser vibrometer measuring the response of the UAS.

through the use of a suction cup. The shaker was set to random excitation with the amplitude set to an appropriate excitation level; in this test case it was $30 \%$ of the maximum gain setting on the amplifier. The amplitude of excitation is chosen to provide sufficient excitation for a strong signal while being small enough not to damage the shaker or aircraft. A signalto-noise ratio of greater than $+5 \mathrm{~dB}$ is desired, which typically produces consistent results from multiple tests. The excitation level chosen should give clear and repeatable test data where the signal is clearly identifiable over the noise floor. The $30 \%$ of the maximum level of the amplifier in this testing provided very repeatable results without the risk of damage to the structure. In other testing configurations the appropriate level can be found by incrementally increasing the amplitude until suitable results are obtained.

It is recommended that, when using this method, the response is measured at grid points over the entire structure. However, this method is very sensitive to grid size since it does not distinguish if it is repeatedly capturing the same mode and so, if the grid is too fine, it could identify multiple points as significant that all correspond to the same response. Therefore, good candidate measurement locations are critical in using this method. The grid needs to be fine enough that all of the modes of interest (i.e., bending, torsion) are captured but not so fine that the same behavior is captured by several 


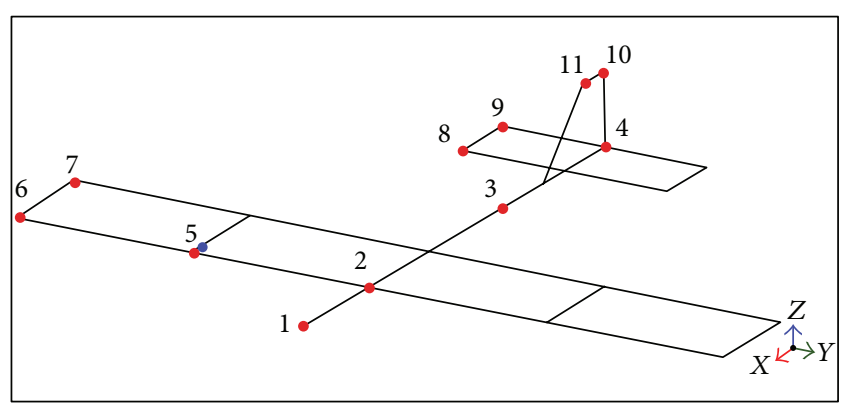

Figure 3: Excitation (blue) and measurement (red) locations for laser vibrometer tests. The airframe is approximated $3.7 \mathrm{~m}$ long and $3.1 \mathrm{~m}$ wide.

locations. A good candidate grid can be established from the structural response of similar structures, finite element results, or previous experience. However, additional points to those that are deemed potentially significant should be included as there could be unexpected participating modes. This method can help capture these unexpected modes that occur due to complex structural interactions or complex structural response. For example, for the airframe example, a midpoint location on the wing must be included or the model cannot predict any nodes along the length of the wing, thereby failing to capture higher order wing bending. To capture wing and tail torsional modes, points on the leading edge and the trailing edge are necessary. In general, the number of candidate locations in any direction determines the order of the polynomial of the shape function in that direction. The best fit shape function is at maximum one order less than the number of nodes. Therefore, the proper number of candidate nodes in orthogonal directions must be chosen to capture all the potential modes of interest. Sound judgment is required to limit the number of candidate locations while still capturing all the motion of interest. This balance helps streamline the procedure to make it most efficient, but the process is valid for any number of candidate locations.

In this study, a set of candidate locations was chosen that covered the structure in a uniform sampling distribution. The total number of sampling points is dependent on the structure and its complexity. A small candidate set may be appropriate for a system that is expected to have a predictable behavior, while a large set would be appropriate for a complex system. In either case, sufficient samples are required to capture both the anticipated and unanticipated modes that may be otherwise missed. In the sUAS study the relatively small number of candidate locations was deemed sufficient to capture the modes of interest and illustrate the methodology. The number and density of the candidate nodes for inclusion are skill that is developed with experience and trial and error. The proper nodal density for the structure is much like a proper finite element mesh; the density required is such that it provides good results without the added cost of excessive node locations.

The first step in the test procedure was to move the laser vibrometer to a measurement location and focus the laser to

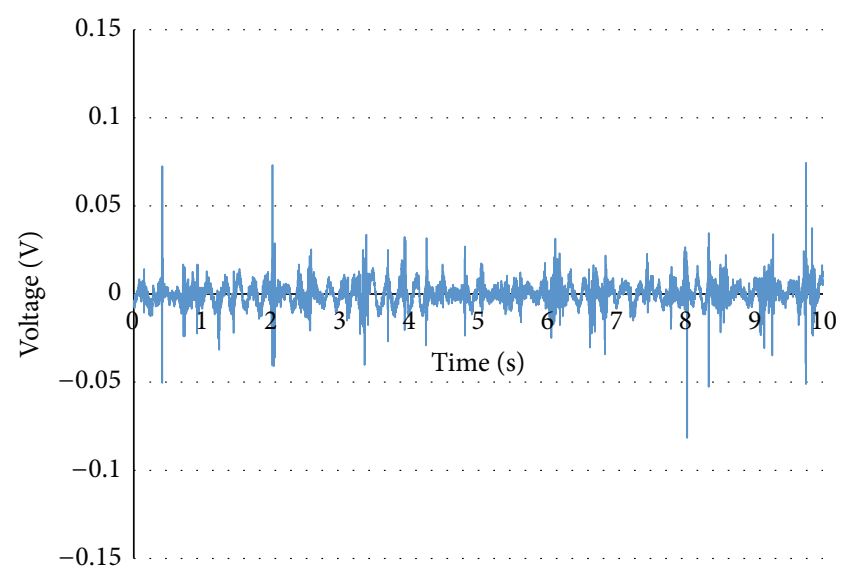

FIGURE 4: A sample of the response measured by the laser vibrometer over a $10 \mathrm{~s}$ period.

get a strong, clear signal. Next, the shaker was activated to vibrate the aircraft with random excitation. LabVIEW Signal Express was used to record the data from the vibrometer over a ten-second period and export it to an Excel worksheet. These steps were repeated until three sets of data were gathered from each measurement location.

\section{Results}

A sample of the data that was gathered is shown in Figure 4 . The graph shows the voltage measurements taken by the laser vibrometer of a test location versus time with the voltage corresponding to amplitude. Postprocessing was manually performed in Microsoft Excel to remove potential outliers caused by signal loss or extraneous noise and then shift the data sets so that they centered about zero. In this case there are voltage spikes that have not been removed or filtered as the authors did not want to overly process the candidate location data to bias the results.

To find an effective measure of the magnitude of vibration at each location, the Root Mean Square (RMS) value for each data set was calculated using

$$
x_{\mathrm{rms}}=\sqrt{\frac{1}{n}\left(x_{1}^{2}+x_{2}^{2}+\cdots+x_{n}^{2}\right)} .
$$

A representative RMS value for each measurement location was then found by calculating the average of the three RMS values from the three data sets at each location, as per Step 4 of the basic procedure. The average RMS values can be seen in Figure 5.

This chart was created using Minitab with $95 \%$ confidence intervals. It can be seen that the nodes are distinctly different and the relatively small size of the error bars indicates that the tests are repeatable.

Once the average RMS values were calculated, the Pareto chart statistical method was used to select the important nodes at which accelerometers need to be placed, as indicated in Step 5. The Pareto chart method was applied to this test by first assuming that all of the motion of the aircraft was 


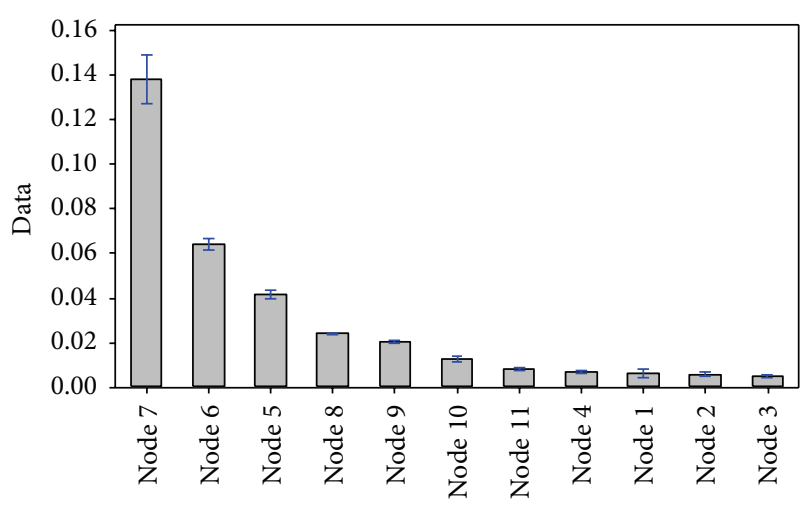

Figure 5: Average RMS values for laser vibrometer measurement locations with $95 \%$ confidence intervals.

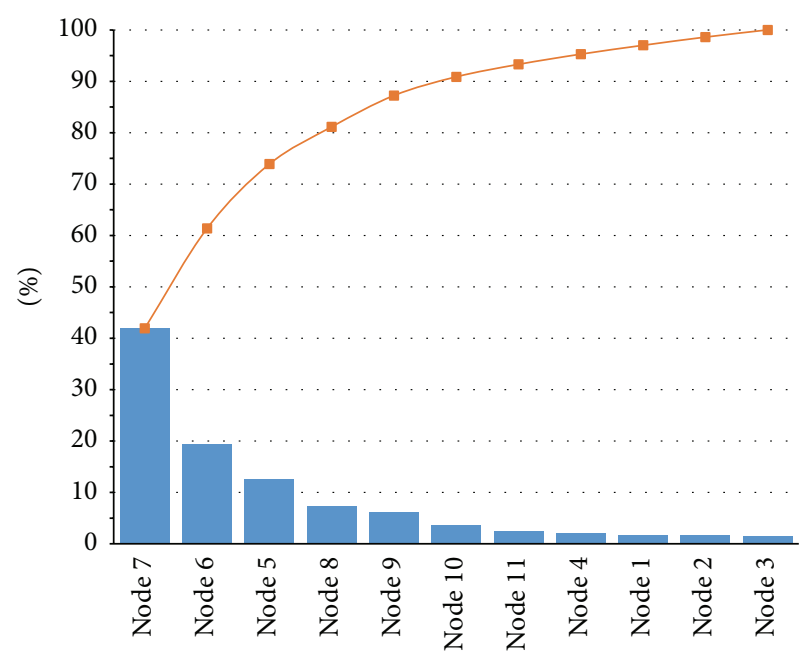

FIGURE 6: Pareto chart for the laser vibrometer test.

captured by the measured locations. The average RMS values were then ordered from largest to smallest and the individual percentages of the total were calculated. These percentages were then summed to find a running cumulative percentage of the total aircraft motion captured. These results were then graphed and are shown in Figure 6. The average RMS values for each of the locations are shown in Table 1. A thorough screening of potential sensor locations must be conducted to help ensure that all the motion of interest is captured. The use of a laser vibrometer makes this process effective due to the efficiency of collecting data.

This chart and table can be used to determine which locations should be measured (Step 6). To capture $75 \%$ of the measured motion, nodes 5,6 , and 7 , which are the nodes on the wings, should be instrumented. Alternatively, to capture $90 \%$ of the measured motion of the aircraft, nodes 8 and 9 on the horizontal stabilizer would have to be instrumented as well. To capture even more motion, nodes 10 and 11 on the vertical stabilizer could be instrumented as well to bring the measured motion up to $95 \%$.
TABLE 1: Data used to create the Pareto chart.

\begin{tabular}{lccc}
\hline Location & Avg RMS & \% of total & Cum. \% \\
\hline Node 7 & 0.1382 & 42 & 42 \\
Node 6 & 0.0640 & 19 & 61 \\
Node 5 & 0.0412 & 13 & 74 \\
Node 8 & 0.0239 & 7 & 81 \\
Node 9 & 0.0201 & 6 & 87 \\
Node 10 & 0.0120 & 4 & 91 \\
Node 11 & 0.0080 & 2 & 93 \\
Node 4 & 0.0065 & 2 & 95 \\
Node 1 & 0.0057 & 2 & 97 \\
Node 2 & 0.0053 & 2 & 99 \\
Node 3 & 0.0046 & 1 & 100 \\
Total & $\mathbf{0 . 3 2 9 4}$ & & \\
\hline
\end{tabular}

\section{Ground Vibration Tests}

GVTs were performed to investigate the validity of the sensor location identification method and to illustrate the different sensor set results (Step 7). Four tests were performed with different levels of instrumentation. These tests included a base test with sensors placed at each measurement location from the laser vibrometer test, a test corresponding to the $95 \%$ Pareto level, one corresponding to $90 \%$, and one corresponding to $75 \%$. The accelerometer locations for each of the tests can be seen in Figure 7. All of the accelerometers were mounted to measure acceleration in the $Z$ direction except for the ones on the vertical stabilizer, which were mounted to measure in the $Y$ direction.

The final step (Step 8) was to conduct a complete modal analysis of the structure. The aircraft was excited at several different locations, all in the $Z$ direction, that are identified by the red dots in Figure 8 and a load cell was attached in line with the shaker's stinger to measure input force.

The accelerometers and excitation devices were routed through a National Instruments Data Acquisition Board that was connected directly to a computer. Data capture and analysis were performed using ModalVIEW, software designed specifically for modal testing and analysis. Information on the equipment that was used is shown in Table 2. The natural frequencies presented are the average of eight tests. These eight tests are comprised of two tests at each of the four excitation locations.

The measurement type was set to FRF-EMA in ModalVIEW for an experimental modal analysis. The sampling rate was left at the default of $1651.61 \mathrm{~Hz}$ with the resolution set to $0.1 \mathrm{~Hz}$. The shaker was activated so that the Super Hauler was excited with random excitation. ModalVIEW was then prompted to record data from the accelerometers and when ModalVIEW was done sampling the shaker was turned off. This was repeated so that two data sets were gathered at each excitation location.

Once all of the vibration data was gathered, analysis was performed using ModalVIEW and followed the steps outlined as follows. First, ModalVIEW automatically generated FRFs from the data for each channel. A curve could then be 
TABLE 2: Test equipment information.

\begin{tabular}{llcc}
\hline Description & Model & Sensitivity & Resolution \\
\hline Uniaxial accelerometer & PCB Piezotronics 352C18 & $10 \mathrm{mV} / \mathrm{g}$ & $0.0005 \mathrm{~g}$ \\
Uniaxial accelerometer & PCB Piezotronics 352C33 & $100 \mathrm{mV} / \mathrm{g}$ & $0.00015 \mathrm{~g}$ \\
Triaxial accelerometer & PCB Piezotronics 356A32 & $100 \mathrm{mV} / \mathrm{g}$ & $0.0003 \mathrm{~g}$ \\
Load cell & PCB Piezotronics 208C02 & $50 \mathrm{mV} / \mathrm{g}$ & - \\
Shaker & The Modal Shop Inc. K2007E01 & - & - \\
Data acquisition board & National Instruments cDAQ-9178 & - & - \\
Data acquisition module & National Instruments 9234 & - & - \\
\hline
\end{tabular}

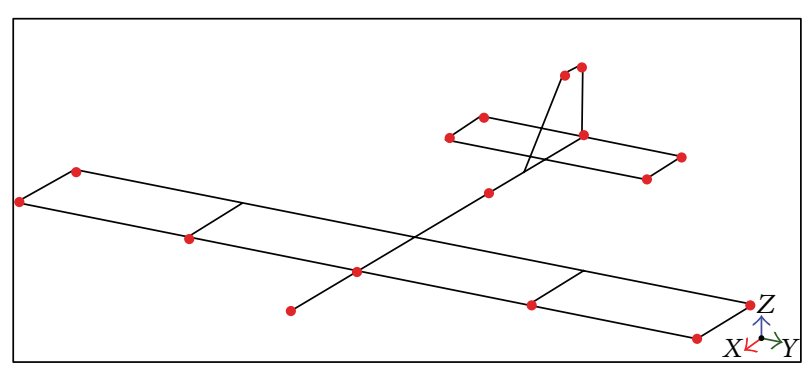

(a)

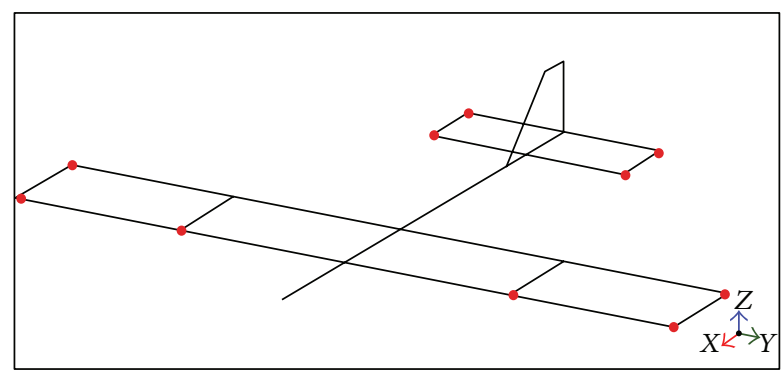

(d)

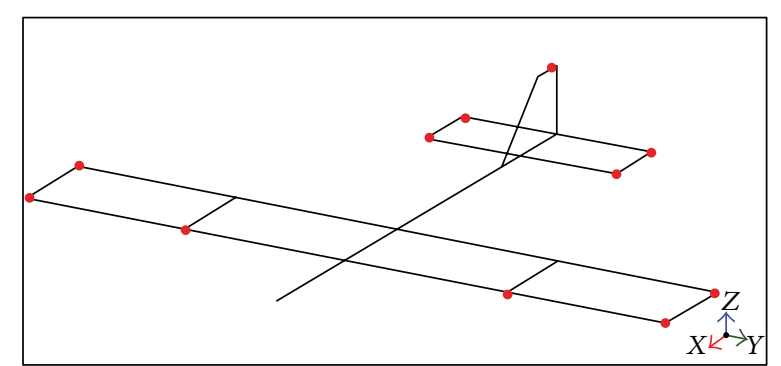

(b)

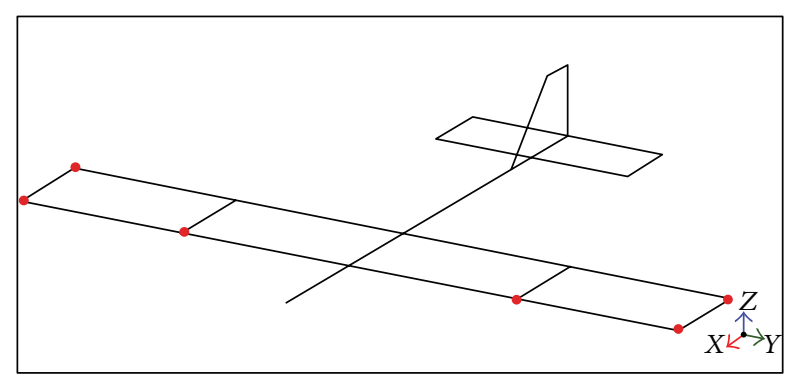

(c)

Figure 7: Clockwise from (a) base test, 95\% test, 75\% test, and 90\% test.

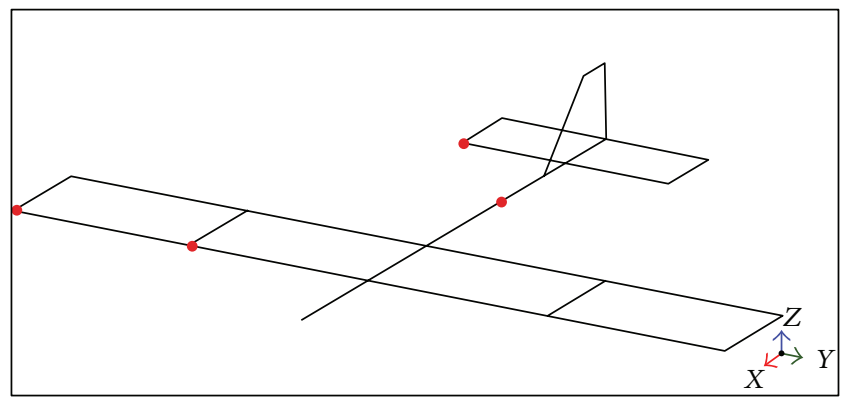

Figure 8: Shaker excitation locations.

fit to the FRF by selecting a frequency range for ModalVIEW to analyze. The peaks within this range indicate the number of modes within that range. This can be seen in Figure 9. The ranges selected varied and depended on the curve. The ranges were chosen so that a good curve fit could be obtained. If there was a good curve fit, the frequencies would then be added to the modal natural frequency list for that test. FRFs

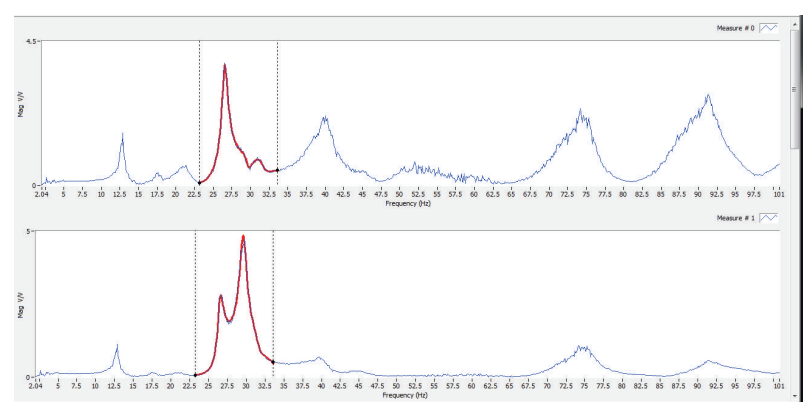

FIGURE 9: Identifying modes from an FRF using ModalVIEW.

were analyzed in this manner until a list of modal natural frequencies was created for each test.

ModalVIEW was then used to build a model of the Super Hauler. The accelerometers were assigned to their respective nodes and degrees of freedom. The modal response of the structure can be animated illustrating the motion of each mode shape at its natural frequency. 
TABLE 3: Summary of natural frequencies.

\begin{tabular}{|c|c|c|c|c|}
\hline Base & $95 \%$ & $90 \%$ & $75 \%$ & Description \\
\hline 12.4 & 12.4 & 12.4 & 12.4 & Mode 1 wing bending \\
\hline 17.3 & 17.5 & 17.5 & 17.5 & $\begin{array}{l}\text { Tail torsion, antisymmetric wingtip } \\
\text { bend }\end{array}$ \\
\hline 20.8 & 20.8 & 20.8 & 21.0 & $\begin{array}{l}\text { Wingtip, wing, tail, HS, VS torsion, } \\
\text { and wingtip bend }\end{array}$ \\
\hline 26.7 & 26.0 & 26.0 & 25.9 & Wing torsion, slight HS bend \\
\hline 28.9 & 28.2 & 28.1 & 28.1 & Symmetric wing torsion \\
\hline 30.2 & 30.8 & 30.8 & 30.7 & $\begin{array}{l}\text { Antisymmetric wingtip bend, tail } \\
\text { wag }\end{array}$ \\
\hline 33.8 & 36.5 & 36.1 & - & HS, VS torsion \\
\hline 39.9 & 39.7 & 39.8 & 39.8 & $\begin{array}{l}\text { HS bend/torsion, mode } 2 \text { wing } \\
\text { bend, and VS torsion }\end{array}$ \\
\hline 45.0 & 44.9 & 44.9 & 44.7 & $\begin{array}{l}\text { Mode } 2 \text { wing bend, HS } \\
\text { bend/torsion, and VS torsion }\end{array}$ \\
\hline 51.4 & 51.0 & 51.7 & 50.6 & Wing torsion, HS bend/torsion \\
\hline 63.7 & 61.1 & 62.9 & 64.1 & $\begin{array}{l}\text { Antisymmetric wing torsion, HS } \\
\text { bend, and fuselage bend }\end{array}$ \\
\hline 72.3 & 73.3 & 72.0 & 71.9 & $\begin{array}{l}\text { Mode } 2 \text { wing bend/torsion opposite, } \\
\text { HS torsion }\end{array}$ \\
\hline 74.4 & 75.9 & 75.9 & 75.8 & $\begin{array}{l}\text { HS torsion, mode } 2 \text { wing bend and } \\
\text { torsion }\end{array}$ \\
\hline
\end{tabular}

A summary of the natural frequencies found and a brief description of the motion observed in the tests can be seen in Table 3 and the corresponding mode shapes can be seen in Figure 10.

It can be seen that all of the natural frequencies that were observed in the base model were also observed in the 95\% test. However, as would make sense from the channels that were removed, any motion in the fuselage was undetectable using the $95 \%$ data. Since there was relatively little activity in the fuselage when compared to the rest of the structure, the inability to observe that motion is fairly insignificant. This test only uses 11 sensors versus 16 sensors for the base test resulting in a $31 \%$ reduction in sensors.

The $90 \%$ test also captured all of the natural frequencies that were seen in the base model. However, any motion in the fuselage or vertical stabilizer was undetectable using the $90 \%$ data. The inability to observe this motion could be acceptable since the fuselage and vertical stabilizer do not experience significant motion and all of the modes were detected. This test uses 10 sensors versus 16, resulting in a $38 \%$ reduction in sensors.

The 75\% test captured almost all of the natural frequencies that were seen in the base model, missing one mode at $33.8 \mathrm{~Hz}$. However, any motion in the fuselage or tail was undetectable in this test. The inability to detect a mode at $33.8 \mathrm{~Hz}$ can be traced to this fact since that mode consists of horizontal and vertical stabilizer motion. This lack of important information leads to the conclusion that this is too much channel reduction and that all of the important surfaces of the aircraft should be instrumented. This test uses 6 sensors versus 16 , resulting in a $63 \%$ reduction in sensors.

It can be seen that, according to this test, $90 \%$ of the motion should be captured by sensors if all of the modes are to be recognized. The values chosen in this case were chosen because they coincide with the significant structural components of the aircraft while resulting in nearly a $40 \%$ reduction in required channels. Since the correct level is case dependent, this value should be left to user discretion.

\section{Conclusion}

A novel modal sensor location identification method was presented. This method was shown to provide a quick, relatively simple, noncontact experimental way to determine important sensor locations through the use of a Pareto chart. This development could be of interest to the vibration community as there has not been any evidence found of a similar method. The laser vibrometer was used to measure the response at several locations along the aircraft and the RMS values were calculated. A Pareto chart was used to identify which of these locations is important to instrument by identifying which locations contribute to most of the motion experienced by the aircraft. This method would be most effectively used by measuring the motion at several locations on the structure and placing sensors at the nodes that capture a certain percentage of the motion that was measured. This provides the user with the flexibility to choose the percentage of motion that is important for that structure.

A study of a small UAS was done on channel reduction and three tests with different levels of channel reduction were performed and analyzed. From these tests it was found that the sensors along the fuselage of the aircraft and on the vertical stabilizer are unnecessary for this testing since all of the modes were still identifiable. The sensors on the wings and horizontal stabilizer were found to be necessary. This conclusion was reached because of the loss of ability to identify a mode involving only horizontal and vertical stabilizer motion when only the wings were instrumented. Using these findings, the number of channels used in this test could be reduced from 16 channels down to 10 channels, which is a $38 \%$ reduction in channels. This reduction not only saves money by using less sensors and supporting equipment, but also saves time that would have been spent on data collection and analysis on the extra channels.

In addition, this experimental procedure can be used in conjunction with numerical simulations for model validation and other sensor placement optimization techniques. The procedure also can augment the evaluation and assessment of their structural behavior of previously constructed structures. Future utilization and expansion of the methodology presented include the correlation of the Pareto chart method to other sensor placement techniques. A mathematical equivalency of the modal participation of the sensor locations could prove the level of motion predicted using the Pareto chart method. This analysis would help verify the methodology but is not required for the practical implementation of the procedure. Also, studies providing additional guidelines for candidate sensor locations could improve the efficacy of the method. The number of grid locations is a challenging issue with the method. The proper candidate set selection is now established using engineering insight on the structure to choose an adequate number of observation points. Tools, 


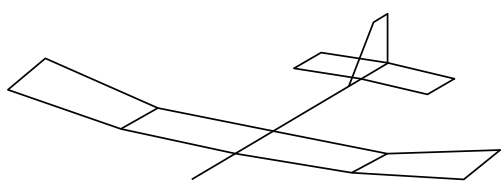

$12.4 \mathrm{~Hz}$

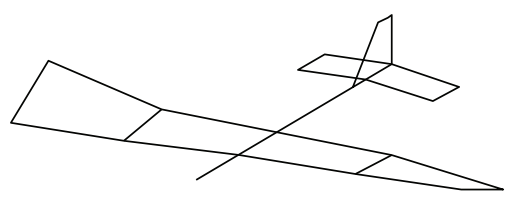

$26.7 \mathrm{~Hz}$

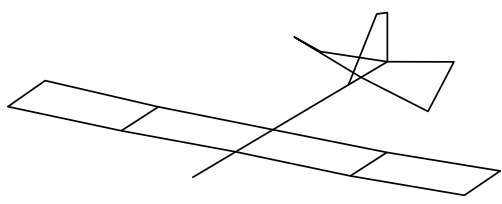

$33.8 \mathrm{~Hz}$

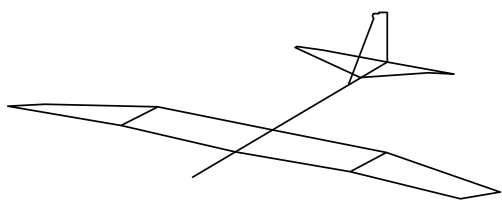

$51.4 \mathrm{~Hz}$

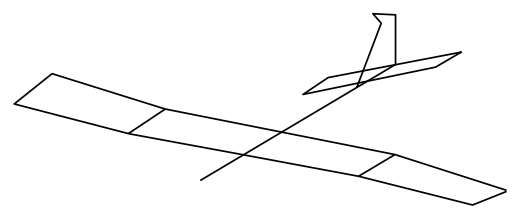

$17.3 \mathrm{~Hz}$

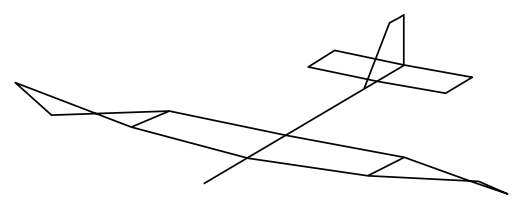

$28.9 \mathrm{~Hz}$

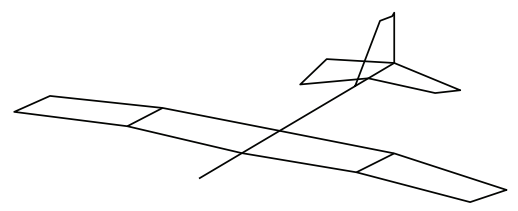

$39.9 \mathrm{~Hz}$

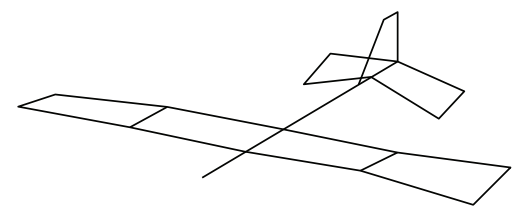

$63.7 \mathrm{~Hz}$

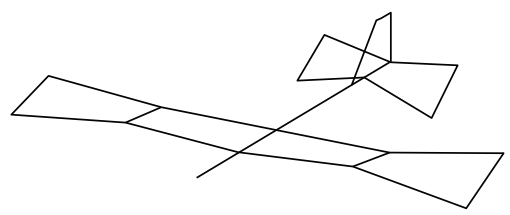

$74.4 \mathrm{~Hz}$

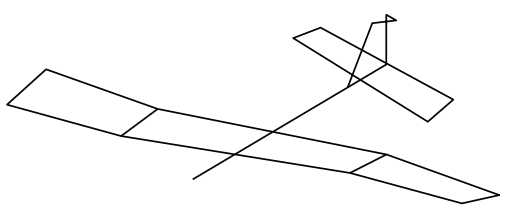

$20.8 \mathrm{~Hz}$

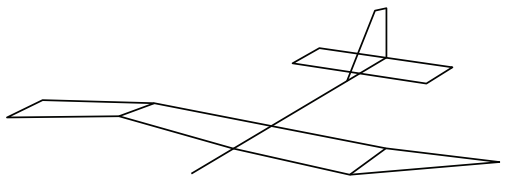

$30.2 \mathrm{~Hz}$

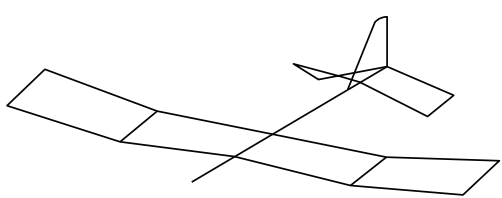

$45.0 \mathrm{~Hz}$

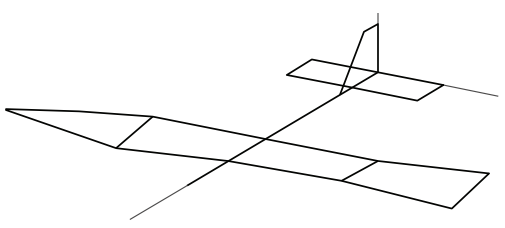

$73.3 \mathrm{~Hz}$

FIgUre 10: Mode shapes obtained from the GVT on the UAS.

such as those used in finite element modeling to establish proper mesh density, may be very useful and provide guidance in selecting the mesh that provides sufficient details without excessive costs in the implementation or utilization. In general, this purely experimental procedure provides an effective method for enhanced modal analysis and can serve as a supplement to the procedures currently engaged in by the modal analysis community.

\section{Conflict of Interests}

The authors declare that there is no conflict of interests regarding the publication of this paper.

\section{Acknowledgments}

This work was supported in part by the Department of Defense Contract no. FA4861-06-C-C006 “Unmanned Aerial
System Remote Sense and Avoid System and Advanced Payload Analysis and Investigation," the Air Force Research Laboratory "MEMS Antenna for Wireless Communications Supporting Unmanned Aerial Vehicles in the Battlefield," and the North Dakota Department of Commerce "UND Center of Excellence for UAV and Simulation Applications."

\section{References}

[1] G. A. Fleming, R. D. Buehrle, and O. L. Storaasli, "Modal analysis of an aircraft fuselage panel using experimental and finite-element techniques," in 3rd International Conference on Vibration Measurements by Laser Techniques: Advances and Applications, vol. 3411 of Proceedings of SPIE, Anacona, Italy, 1998.

[2] T. G. Carne and C. R. Dohrmann, "A modal test design strategy for model correlation," in Proceedings of the 13th International Modal Analysis Conference (IMAC '95), Nashville, Tenn, USA, 1995. 
[3] D. C. Kammer, "Sensor set expansion for modal vibration testing," Mechanical Systems and Signal Processing, vol. 19, no. 4, pp. 700-713, 2005.

[4] A.-P. Cherng, "Optimal sensor placement for modal parameter identification using signal subspace correlation techniques," Mechanical Systems and Signal Processing, vol. 17, no. 2, pp. 361378, 2003.

[5] C. Stephan, "Sensor placement for modal identification," Mechanical Systems and Signal Processing, vol. 27, no. 1, pp. 461470, 2012.

[6] D. C. Kammer and M. L. Tinker, "Optimal placement of triaxial accelerometers for modal vibration tests," Mechanical Systems and Signal Processing, vol. 18, no. 1, pp. 29-41, 2004.

[7] M. Stabb and P. Blelloch, "A genetic algorithm for optimally selecting accelerometer locations," in Proceedings of the 13th International Modal Analysis Conference (IMAC-XIII), Nashville, Tenn, USA, 1995.

[8] C. He, J. Xing, J. Li, Q. Yang, R. Wang, and X. Zhang, "A new optimal sensor placement strategy based on modified modal assurance criterion and improved adaptive genetic algorithm for structural health monitoring," Mathematical Problems in Engineering, vol. 2015, Article ID 626342, 10 pages, 2015.

[9] L. He, J. Lian, B. Ma, and H. Wang, "Optimal multiaxial sensor placement for modal identification of large structures," Structural Control and Health Monitoring, vol. 21, no. 1, pp. 6179, 2014.

[10] W. Liu, W.-C. Gao, Y. Sun, and M.-J. Xu, "Optimal sensor placement for spatial lattice structure based on genetic algorithms," Journal of Sound and Vibration, vol. 317, no. 1-2, pp. 175-189, 2008.

[11] T. H. Yi, H. N. Li, and M. Gu, "Optimal sensor placement for health monitoring of high-rise structure based on genetic algorithm," Mathematical Problems in Engineering, vol. 2011, Article ID 395101, 12 pages, 2011.

[12] E. Balmes, "Orthogonal maximum sequence sensor placements algorithms for modal tests, expansion and visibility," in Proceedings of the 23rd International Modal Analysis Conference (IMACXXIII '05), Orlando, Fla, USA, January 2005.

[13] C. Papadimitriou, "Pareto optimal sensor locations for structural identification," Computer Methods in Applied Mechanics and Engineering, vol. 194, no. 12-16, pp. 1655-1673, 2005.

[14] D. C. Kammer, "Sensor placement for on-orbit modal identification and correlation of large space structures," Journal of Guidance, Control, and Dynamics, vol. 14, no. 2, pp. 251-259, 1991.

[15] P. H. Kirkegaard and R. Brincker, "On the optimal location of sensors for parametric identification of linear structural systems," Mechanical Systems and Signal Processing, vol. 8, no. 6, pp. 639-647, 1994.

[16] J. E. T. Penny, M. I. Friswell, and S. D. Garvey, "Automatic choice of measurement locations for dynamic testing," AIAA Journal, vol. 32, no. 2, pp. 407-414, 1994.

[17] P. C. Shah and F. E. Udwadia, "Methodology for optimal sensor locations for identification of dynamic systems," Journal of Applied Mechanics, Transactions ASME, vol. 45, no. 1, pp. 188196, 1978.

[18] F. E. Udwadia, "Methodology for optimum sensor locations for parameter identification in dynamic systems," Journal of Engineering Mechanics, vol. 120, no. 2, pp. 368-390, 1994.

[19] R. G. Cobb and B. S. Liebst, "Sensor placement and structural damage identification from minimal sensor information," AIAA Journal, vol. 35, no. 2, pp. 369-374, 1997.
[20] E. Heredia-Zavoni, R. Montes-Iturrizaga, and L. Esteva, "Optimal instrumentation of structures on flexible base for system identification," Earthquake Engineering and Structural Dynamics, vol. 28, no. 12, pp. 1471-1482, 1999.

[21] P. M. Hemez and C. Farhat, "An energy based optimum sensor placement criterion and its application to structural damage detection," in Proceedings of the 12th International Modal Analysis Conference, Honolulu, Hawaii, USA, 1994.

[22] Z. Y. Shi, S. S. Law, and L. M. Zhang, "Optimum sensor placement for structural damage detection," Journal of Engineering Mechanics, vol. 126, no. 11, pp. 1173-1179, 2000.

[23] H. Sohn and K. H. Law, "A Bayesian probabilistic approach for structure damage detection," Earthquake Engineering and Structural Dynamics, vol. 26, no. 12, pp. 1259-1281, 1997.

[24] A. Rao, K. Lakshmi, and S. Krishnakumar, "A generalized optimal sensor placement technique for structural health monitoring and system identification," Procedia Engineering, vol. 86, pp. 529-538, 2014.

[25] T.-H. Yi, H.-N. Li, and X.-D. Zhang, "Health monitoring sensor placement optimization for canton Tower using immune monkey algorithm," Structural Control and Health Monitoring, vol. 22, no. 1, pp. 123-138, 2015.

[26] T.-H. Yi, H.-N. Li, and M. Gu, "Optimal sensor placement for structural health monitoring based on multiple optimization strategies," Structural Design of Tall and Special Buildings, vol. 20, no. 7, pp. 881-900, 2011.

[27] T.-H. Yi, H.-N. Li, and M. Gu, "A new method for optimal selection of sensor location on a high-rise building using simplified finite element model," Structural Engineering and Mechanics, vol. 37, no. 6, pp. 671-684, 2011.

[28] D. S. Li, H. N. Li, and C. P. Fritzen, "The connection between effective independence and modal kinetic energy methods for sensor placement," Journal of Sound and Vibration, vol. 305, no. 4-5, pp. 945-955, 2007.

[29] N. Debnath, A. Dutta, and S. K. Deb, "Placement of sensors in operational modal analysis for truss bridges," Mechanical Systems and Signal Processing, vol. 31, pp. 196-216, 2012.

[30] J. L. Beck and L. S. Katafygiotis, "Updating models and their uncertainties. I: Bayesian statistical framework," Journal of Engineering Mechanics, vol. 124, no. 4, pp. 455-461, 1998.

[31] J. L. Beck and K.-V. Yuen, "Model selection using response measurements: bayesian probabilistic approach," Journal of Engineering Mechanics, vol. 130, no. 2, pp. 192-203, 2004.

[32] L. S. Katafygiotis, C. Papadimitriou, and H.-F. Lam, "A probabilistic approach to structural model updating," Soil Dynamics and Earthquake Engineering, vol. 17, no. 7-8, pp. 495-507, 1998.

[33] C. Papadimitriou, J. L. Beck, and S.-K. Au, "Entropy-based optimal sensor location for structural model updating," Journal of Vibration and Control, vol. 6, no. 5, pp. 781-800, 2000.

[34] K.-V. Yuen and S.-C. Kuok, "Efficient Bayesian sensor placement algorithm for structural identification: a general approach for multi-type sensory systems," Earthquake Engineering and Structural Dynamics, vol. 44, pp. 757-774, 2015.

[35] R. B. Jenal, W. J. Staszewski, A. Klepka, and T. Uhl, "Structural damage detection using laser vibrometers," in Proceedings of the 2nd International Symposium on NDT in Aerospace, Hamburg, Germany, 2010.

[36] V. Pareto, Manuale di Economia Politician, AM Kelley, New York, NY, USA, 1971.

[37] A. K. Keshri, R. Patel, and A. Agarwal, "Comprehensive process maps to synthesize high density plasma sprayed aluminum 
oxide composite coatings with varying carbon nanotube content," Surface and Coatings Technology, vol. 205, no. 3, pp. 690702, 2010.

[38] A. Jayswal, X. Li, A. Zanwar, H. H. Lou, and Y. Huang, "A sustainability root cause analysis methodology and its application," Computers and Chemical Engineering, vol. 35, no. 12, pp. 27862798, 2011.

[39] C. C. Heyde and E. Seneta, Statisticians of the Centuries, Springer, New York, NY, USA, 2001.

[40] J. S. Lawson and J. Erjavec, Modern Statistics for Engineering and Quality Improvement, Duxbury, Pacific Grove, Calif, USA, 2001.

[41] J. Simisiriwong, Structural testing of an ultralight UAV composite wing and fuselage [M.S. thesis], Mississippi State University, 2009.

[42] D. J. Ewins, Modal Testing: Theory, Practice, and Application, Research Studies Press, Baldock, UK, 2nd edition, 2000.

[43] K. J. Lemler and W. H. Semke, "Structural analysis of the effects of wing payload pods on small UAS," in Proceedings of the 30th International Modal Analysis Conference (IMAC-XXX '12), Jacksonville, Fla, USA, February 2012.

[44] S. Perinpanayagam and D. J. Ewins, "Free-free, fixed or other test boundary conditions for the best modal test?" in Proceedings of the 21st International Modal Analysis Conference (IMAC '03), Kissimmee, Fla, USA, 2003. 

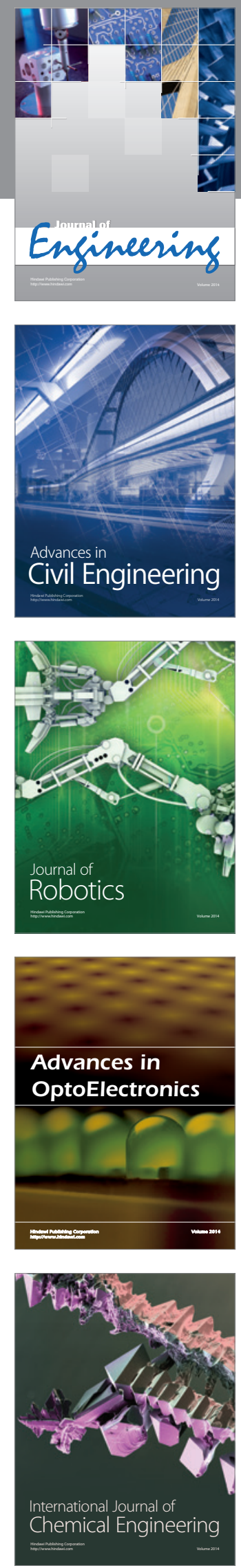

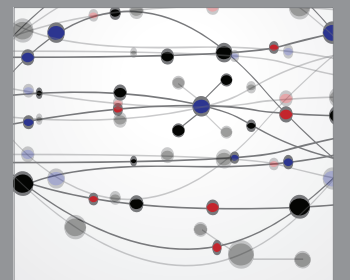

The Scientific World Journal
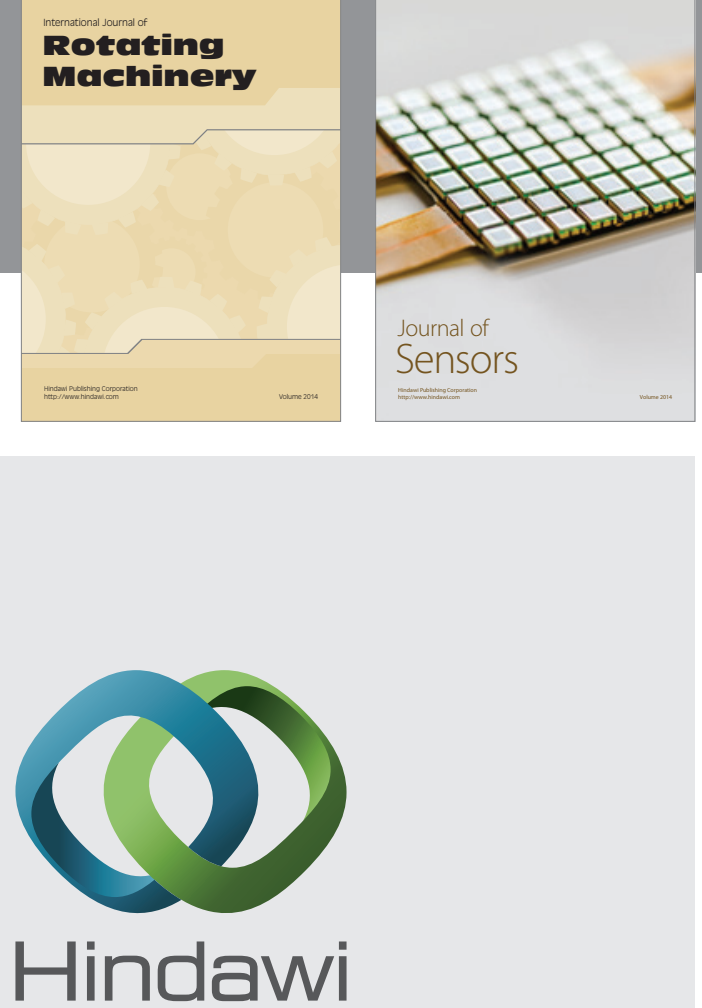

Submit your manuscripts at http://www.hindawi.com
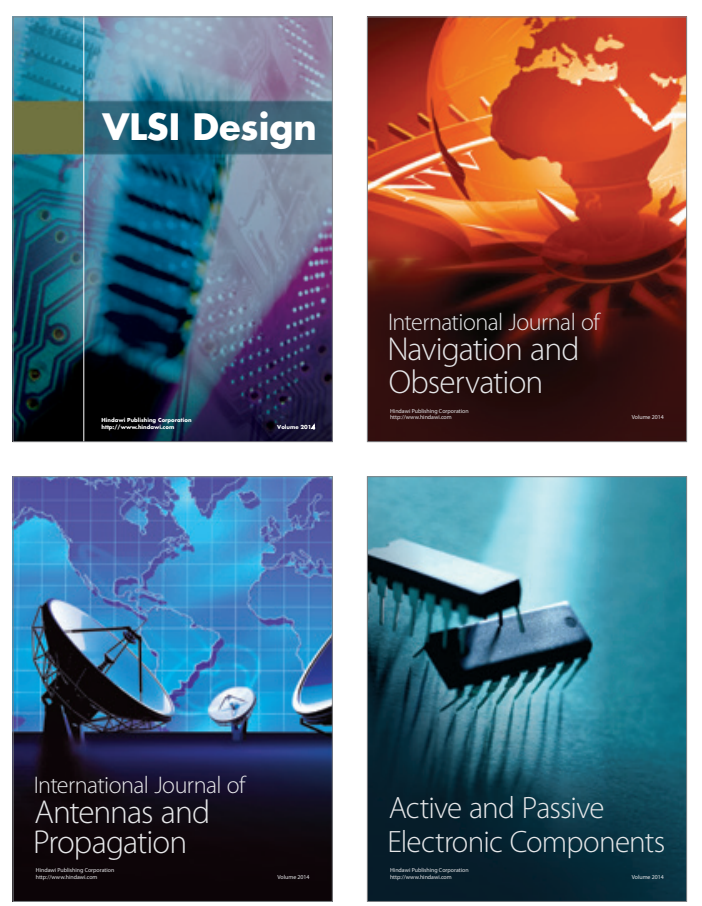
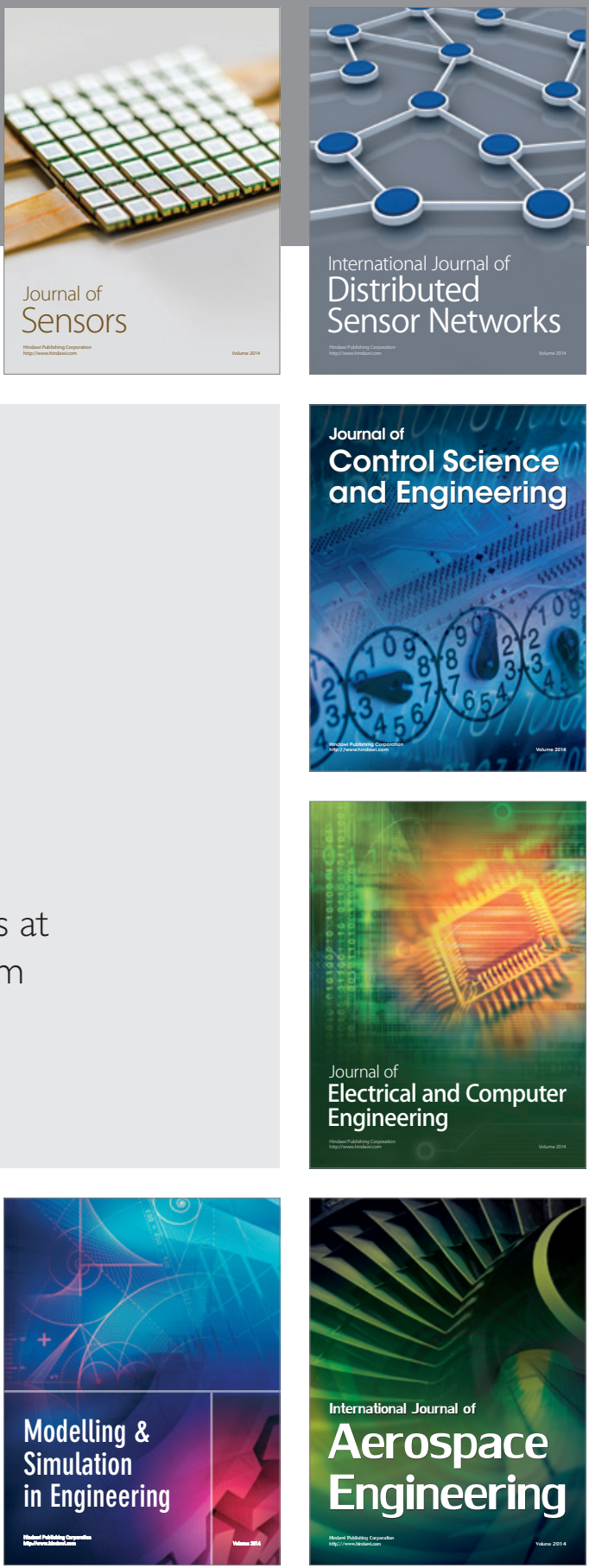

Journal of

Control Science

and Engineering
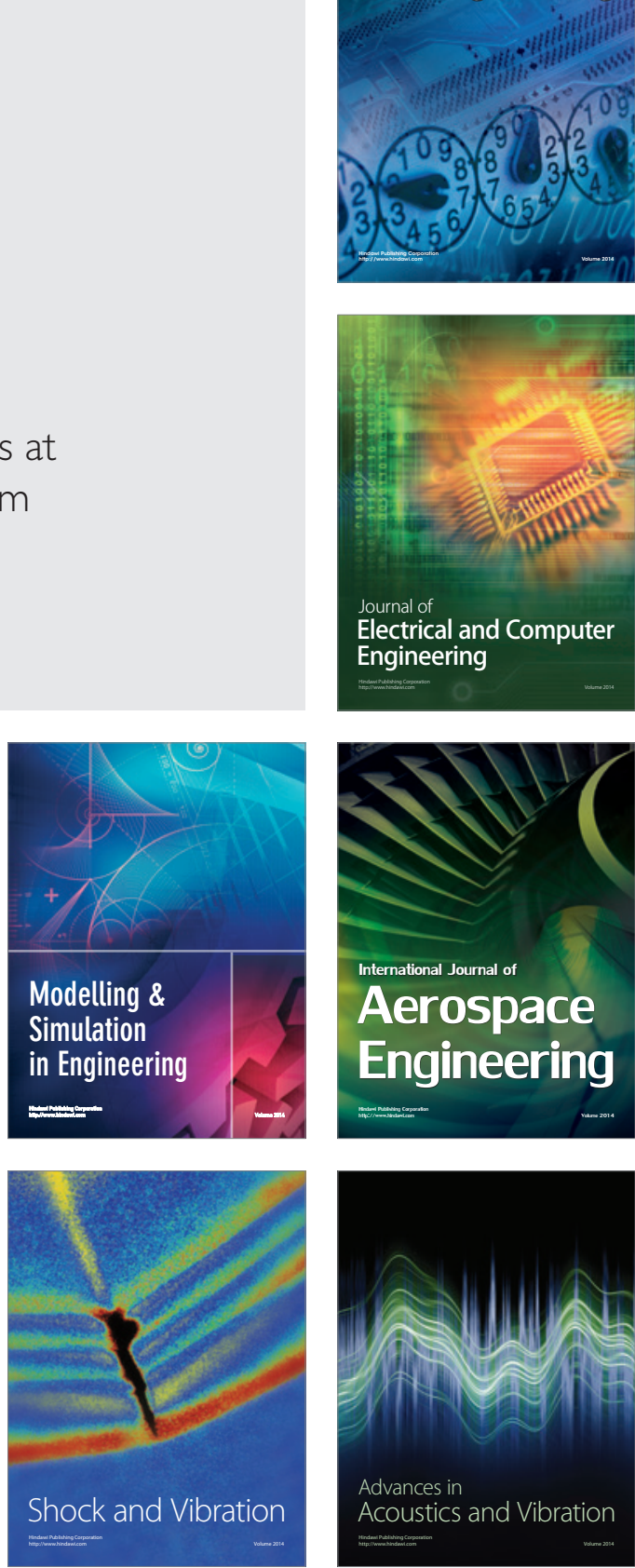